\title{
QUALITY METRICS EVALUATION OF HYPERSPECTRAL IMAGES
}

\author{
A. K. Singh ${ }^{\text {a, }}$, H. V. Kumar ${ }^{\text {d }}$, G. R. Kadambi ${ }^{\text {b }}$, J. K. Kishore ${ }^{\text {a }}$, J. Shuttleworth ${ }^{\text {c }}$, J. Manikandan ${ }^{\text {d }}$ \\ ${ }^{a}$ ISRO Satellite Centre, Bangalore, India - (arksingh, jkk)@isac.gov.in \\ ${ }^{b}$ Dept. of Research, M. S. Ramaiah School of Advanced Studies, Bangalore, India - govind@msrsas.org \\ ${ }^{\mathrm{c}}$ Faculty of Engineering and Computing, Coventry University, UK - csx239@ coventry.ac.uk \\ ${ }^{\mathrm{d}}$ PES Institute of Technology, Bangalore, India - vinod.hosakote@gmail.com, manikandanj@pes.edu
}

\section{Commission VI, WG VI/4}

KEY WORDS: Classification, Evaluation, Hyperspectral, k-means Clustering, Principal Component Analysis, Segmentation.

\begin{abstract}
:
In this paper, the quality metrics evaluation on hyperspectral images has been presented using k-means clustering and segmentation. After classification the assessment of similarity between original image and classified image is achieved by measurements of image quality parameters. Experiments were carried out on four different types of hyperspectral images. Aerial and spaceborne hyperspectral images with different spectral and geometric resolutions were considered for quality metrics evaluation. Principal Component Analysis (PCA) has been applied to reduce the dimensionality of hyperspectral data. PCA was ultimately used for reducing the number of effective variables resulting in reduced complexity in processing. In case of ordinary images a human viewer plays an important role in quality evaluation. Hyperspectral data are generally processed by automatic algorithms and hence cannot be viewed directly by human viewers. Therefore evaluating quality of classified image becomes even more significant. An elaborate comparison is made between k-means clustering and segmentation for all the images by taking Peak Signal-to-Noise Ratio (PSNR), Mean Square Error (MSE), Maximum Squared Error, ratio of squared norms called L2RAT and Entropy. First four parameters are calculated by comparing the quality of original hyperspectral image and classified image. Entropy is a measure of uncertainty or randomness which is calculated for classified image. Proposed methodology can be used for assessing the performance of any hyperspectral image classification techniques.
\end{abstract}

\section{INTRODUCTION}

Hyperspectral images contain information as a set of hundreds of contiguous spectral wavelength bands. The spectral bandwidths of these images are in the order of 5-10nm (Zhang et al., 2001). A true color image will have only three bands red, green, and blue. Even high resolution optical images capture only color information of the objects. The color information is similar for most of the objects. Hence capturing information in the various bands of the spectrum can be used for better classification (Tarabalka et al., 2010). The composition of an object will have unique spectral characteristics that can be used to identify it, which is also known as the concept of spectroscopy.

Hyperspectral remote sensing combines images and spectroscopy in a single system. Some of the major challenges in processing hyperspectral images are:

1. Heavy computational load in processing hyperspectral images due to a large number of bands.

2. Low resolution of satellite images.

We overcome the first challenge by reducing the numbers of bands. Low resolution of image is a serious drawback in identifying the objects.

Each pixel of a hyperspectral image has a unique signature that represents different materials. Hyperspectral image contains up to two hundred or more contiguous spectral wavelength bands, but a few numbers of bands can explain the vast majority of the information. Hence, hyperspectral images are transformed into lower dimensions. For this purpose dimension reduction techniques are used that preserves the main features of the original data by eliminating data redundancy. Principal Component Analysis (PCA) is one of the data reduction techniques often used while analyzing hyperspectral data (Green et al., 1988). PCA is used to reduce the dimensionality of hyperspectral image, while retaining all important features, resulting in reduced complexity and time taken for the further processing. PCA considers all possible projections of the data and chooses the projection with the greatest variance in the first component the second greatest in the second component, and so on.

Classification concepts are important in the design of computerized information processing systems for many applications such as remote sensing and medical diagnosis. A pattern class is a category determined by some common attributes. In remote sensing, spectral reflectances are measured and used as feature to determine the various classes in a given scene. Classification algorithms are often grouped into supervised and unsupervised approaches. When a classifier is given a set of patterns with known classes and is asked to classify a new pattern based on the information learnt during training, it is called a supervised classification. In unsupervised classification, the number of classes is not known a priori. An unsupervised classifier utilizes only the information contained in the measurement data and does not need any training data for each class. It only groups the data into classes which show

\footnotetext{
* Corresponding author. This is useful to know for communication with the appropriate person in cases with more than one author.
} 
similar behaviour in the multidimensional space of observed feature vectors. Thus, classification is achieved by clustering the measured feature vectors, and designating each distinct cluster as a class. Unsupervised classifiers are also known as clustering algorithms.

To evaluate the quality of classified hyperspectral images there are a very few papers. In the area of evaluation of compression losses for hyperspectral images, Christophe et al. (2005) has suggested some of the parameters. It is well known that In the case of normal images, as the end user, a human viewer plays an important role in quality evaluation. Hyperspectral data are generally processed by automatic algorithms and hence cannot be viewed directly by human viewers. Therefore evaluating quality of classified image becomes even more significant.

We have conducted experiments on four different types of hyperspectral images. Aerial and spaceborne hyperspectral images with different spectral and geometric resolutions were considered for quality metrics evaluation. We have considered unsupervised classification algorithms for the simple reason of implementing it on any hyperspectral image without having to worry of training data sets. Here, the quality metrics evaluation on hyperspectral images has been presented using k-means clustering and segmentation. After classification the assessment of similarity between original image and classified image is achieved by measurements of image quality parameters.

\section{PRINCIPAL COMPONENTS ANALYSIS}

PCA transforms an image to a new coordinate system. PCA uses the eigenvectors $(\mathrm{V})$ resulted during eigen decomposition as in equation (1).

$$
\sum=V \Lambda V^{-1}
$$

Where $\Sigma$ is the covariance matrix and $\Lambda$ is a diagonal matrix which contains the eigenvalues corresponding to $\mathrm{V}$, representing new coordinate basis for the image (Phillips et al., 2009).

\section{SEGMENTATION}

Segmentation subdivides an image into its constituent regions or objects (Gonzalez et al., 2009). Monochrome image segmentation is generally based on image intensity values such as discontinuity and similarity. In the first approach partition of an image is based on abrupt changes in intensity like edges. In the second a partitioning of an image is based on the regions that are similar by using a set of predefined criteria. Edge detection is the very common approach for detecting discontinuities in intensity values. These discontinuities are detected by using first- and second-order derivatives (Gonzalez et al., 2009).

Sobel operator is suitable for edge detection because edges are extracted with greater accuracy. Sobel edge detector finds edges using the Sobel approximation to the derivatives. In marker controlled watershed segmentation, sobel operator is used to distinct the edge of the object (Kaur et al., 2011). The sobel masks in matrix form are given in equation (2) and equation (3). Flow chart shown in Figure 1 generates metric evaluation using segmentation technique.

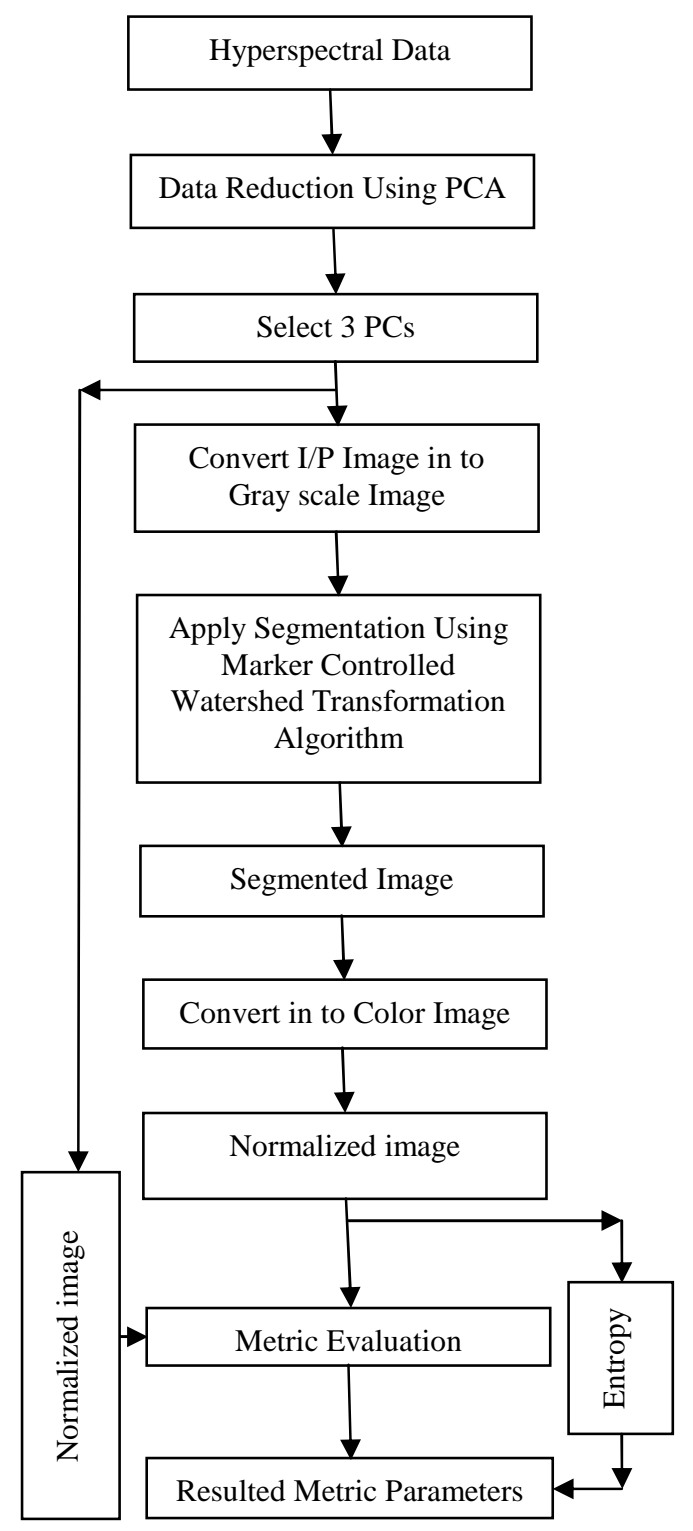

Figure 1. Flow chart for metric evaluation using segmentation

$$
\begin{aligned}
& M x=\left[\begin{array}{ccc}
-1 & 0 & +1 \\
-2 & 0 & +2 \\
-1 & 0 & +1
\end{array}\right] \\
& M y=\left[\begin{array}{ccc}
+1 & +2 & +1 \\
0 & 0 & 0 \\
-1 & -2 & -1
\end{array}\right]
\end{aligned}
$$

Watershed segmentation is a gradient-based technique which considers the gradient map of the image as a relief map. It segments the image as a dam. The segmented regions are called catchment basins. It is suitable for the images that have higher intensity value. To control over segmentation, marker controlled watershed segmentation is used (Acharjya et al., 2012). 


\section{K-MEANS CLUSTERING}

Clustering means the grouping of similar samples in each group. Clustering classifies the pixels with similar characteristics in a cluster. K-means clustering partitions $n$ observations into $\mathrm{k}$ clusters where each observation belongs to the cluster with the nearest mean (Chandhok et al., 2011).

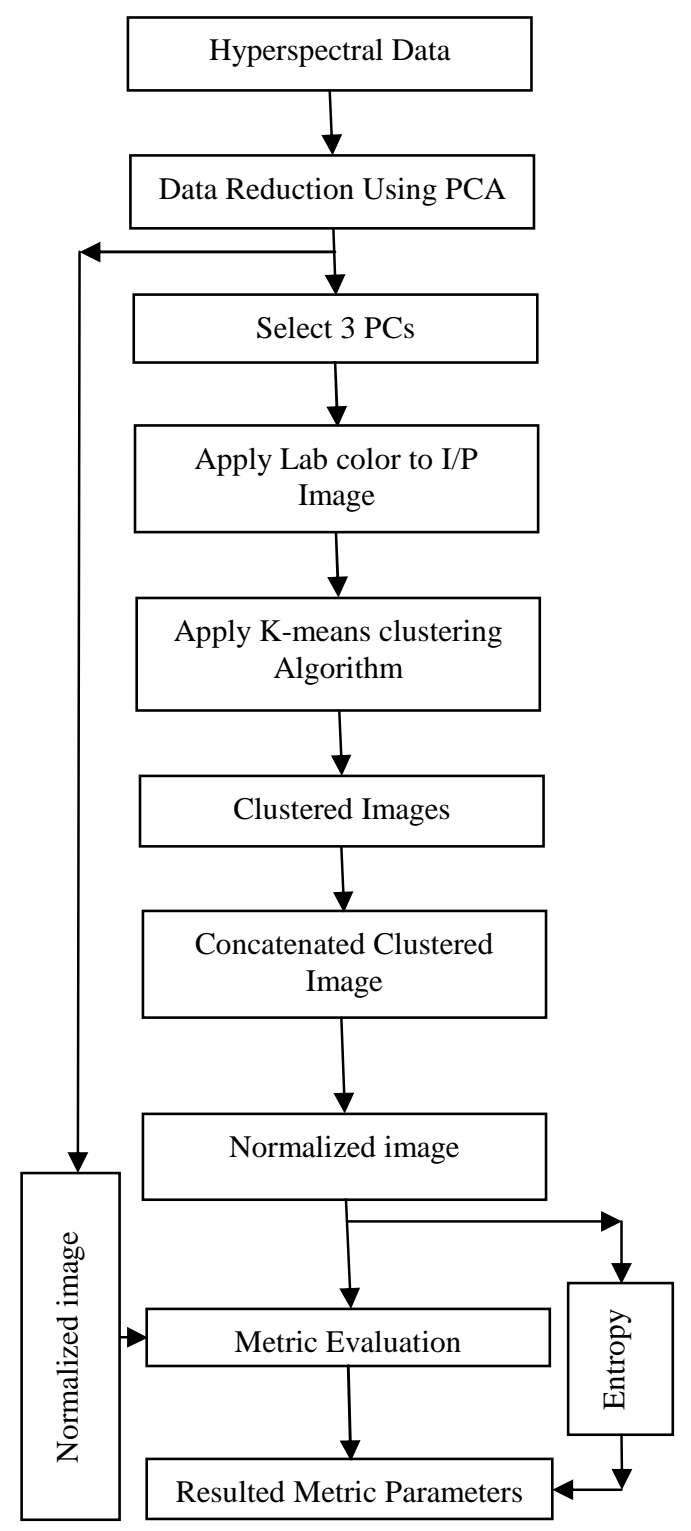

Figure 2. Flow chart for metric evaluation using K-means clustering

K-means takes a simple way for classifying a data set in a certain number of clusters (take $\mathrm{k}$ clusters) fixed a priori. Define $\mathrm{k}$ centroids. Take each point of data set and attach it to the nearest centroid. The pixel with shortest distance moves to a particular cluster. This distance function may be a simple Euclidean function. Ones all point are completed, that indicates completion of the first step and an early grouping is done. Then the centroid is re-estimated. Again each pixel will be compared to all centroids. The process continuous until the center converges. K-Means method is numerical, unsupervised, nondeterministic and iterative (Chandhok et al., 2011). Flow chart shown in Figure 2 generates metric evaluation using K-means clustering.

\section{MEAN SQUARE ERROR}

The Mean Square Error (MSE) as in equation (4) represents the cumulative squared error between the compressed and the original image. Lesser the value of MSE represents lower the error (Christophe et al., 2005).

$$
M S E=\frac{\sum_{x, y, \lambda}\left[I_{1(x, y, \lambda)}-I_{2(x, y, \lambda)}\right]^{2}}{n_{x} n_{y} n_{\lambda}}
$$

Where $I_{1(x, y, \lambda)}$ and $I_{2(x, y, \lambda)}$ are the value from the column $x$ of rows $y$ in the spectral band $\lambda$ in the input images $I_{1}$ and $I_{2}$.

\section{PEAK SIGNAL TO NOISE RATIO}

The Peak Signal to Noise Ratio (PSNR) computes the peak signal-to-noise ratio, in decibels, between two images. This ratio is used for quality measurement between the original and the compressed image. Higher PSNR represents better the quality of the compressed or reconstructed image. PSNR is computed using the equation (5). Here $\mathrm{R}$ is the maximum fluctuation in the input image data type.

$$
P S N R=10 \log _{10}\left(\frac{R^{2}}{M S E}\right)
$$

\section{L2RAT}

L2RAT is the ratio of the squared norm of the image approximation to the input image.

\section{ENTROPY}

Entropy is a statistical measure of randomness that can be used to characterize the texture of the input image. Entropy is defined as in equation (6)

$$
\text { Entropy }=-\sum_{i} \sum_{j} p(i, j) \times \log _{2} p(i, j)
$$

Where $\mathrm{p}$ contains the histogram counts.

\section{EXPERIMENTAL DATA}

In our experiments we have used four numbers of hyperspectral remote sensing data sets from aerial and space born sensors having spatial resolution right from 1.3 meter to 500meter and spectral resolution from 224bands to 64bands.

- First data set: Hydice Washington-DC hyperspectral image was taken on August 23, 1995 (Courtesy: MultiSpec). Due to data handling constraints we have considered 100 rows, 100 columns and 191 spectral bands for processing.

- Second data set: Andaman scene has been taken by Indian satellite IMS-1. The spatial resolution of HySI is about 500 metres. (Courtesy: Bhuvan, NRSC, ISRO, Hyderabad, INDIA). 
- Third data set: Pavia University area scene was acquired by the ROSIS sensor with a spatial resolution of $1.3 \mathrm{~m}$ per pixel (Tarabalka et al., 2010). Pavia University image is $610 \times 340$ pixels and 103 bands.

- $\quad$ Fourth Data Set: Indian Pines image acquired by the AVIRIS sensor is of $145 \times 145$ pixels and 200 bands (Tarabalka et al., 2010) with spatial resolution of 20m/pixel(Courtesy: MultiSpec).

\section{RESULTS AND DISCUSSION}

In our experiments we have processed four hyperspectral images of different spectral and geometric resolutions having different working platforms such as aerial and spaceborn. We have considered image sizes of 100pixels X 100pixels and all spectral bands of respective sensors excluding water absorption bands. We have investigated k-means clustering and segmentation techniques on all these images and generated metric comparisons using PSNR, MSE, L2RAT and Entropy. Flow chart shown in Figure 1 generates metric evaluation using segmentation technique. Similarly flow chart for metric evaluation using K-means clustering is shown in Figure 2. Number of clusters for K-means clustering has been accounted after iterative simulations and getting most appropriate numbers of clusters for the respective scenes. We have used PCA for data reduction techniques which ultimately reduces the number of effective variables resulting in saving processing time while applying K-means clustering and segmentation techniques.

To perform PCA the covariance matrix of all the hyperspectral images were computed. In next step by considering eigen values corresponding eigen vectors were derived. Dimensionality reduced concatenated image consists of three numbers of principal components. These reconstructed images were used for processing K-means clustering and segmentation.

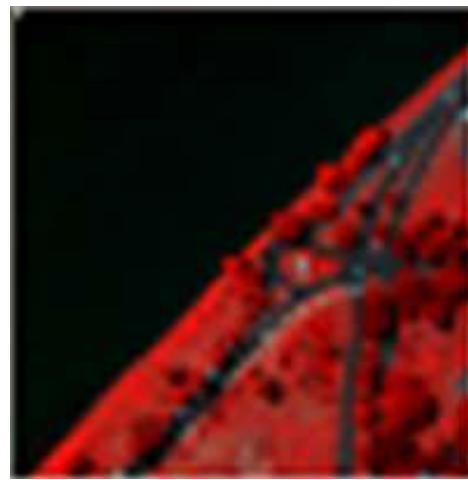

Figure 3. A portion of Hydice Washington-DC hyperspectral image
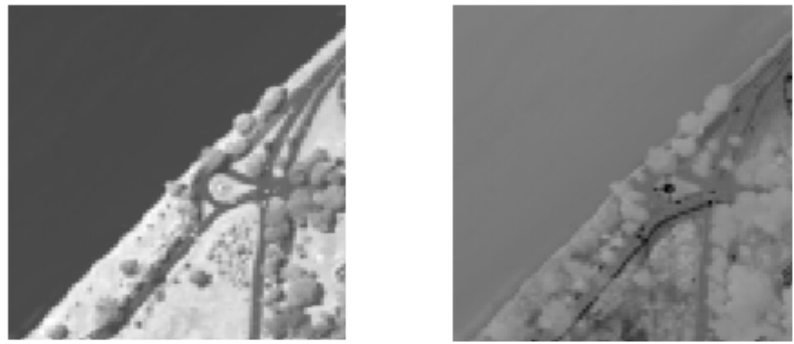

Figure 4. First two Principal Components (PCs) of a portion of Hydice Washington-DC hyperspectral image

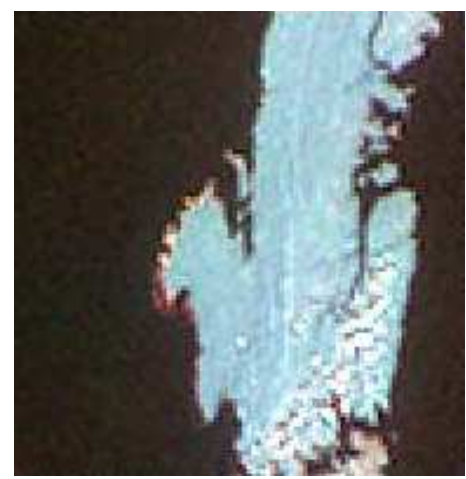

Figure 5. A portion of Andaman (IMS1) hyperspectral image
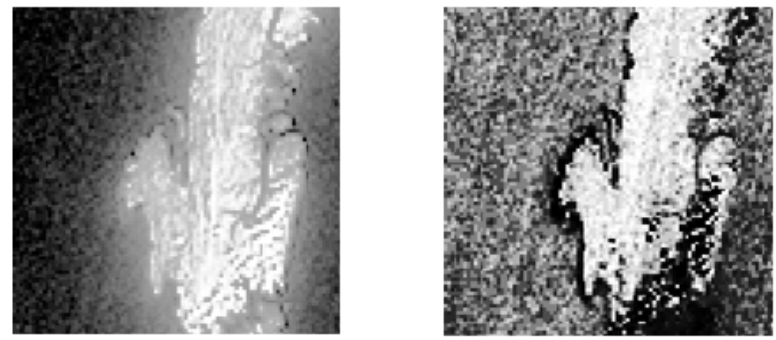

Figure 6. First two Principal Components of a portion of Andaman (IMS1) hyperspectral image

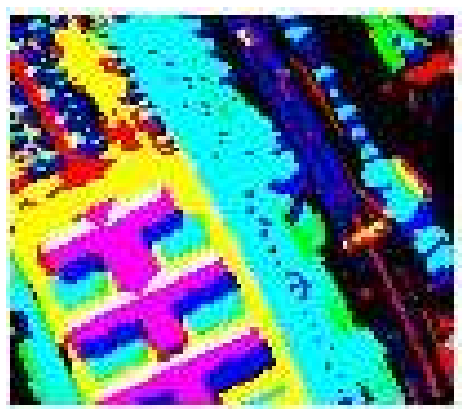

Figure 7. Concatenated image consists of three numbers of principal components of a portion of Pavia University 

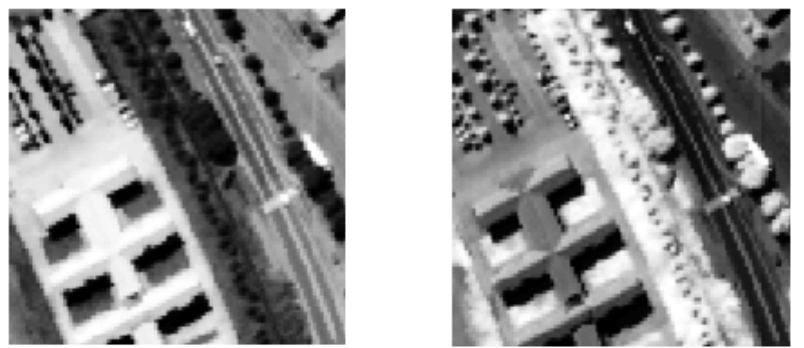

Figure 8. First two PCs of a portion of Pavia University hyperspectral image

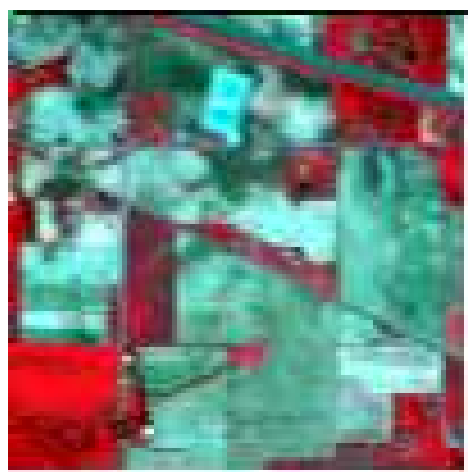

Figure 9. Indian pines hyperspectral image
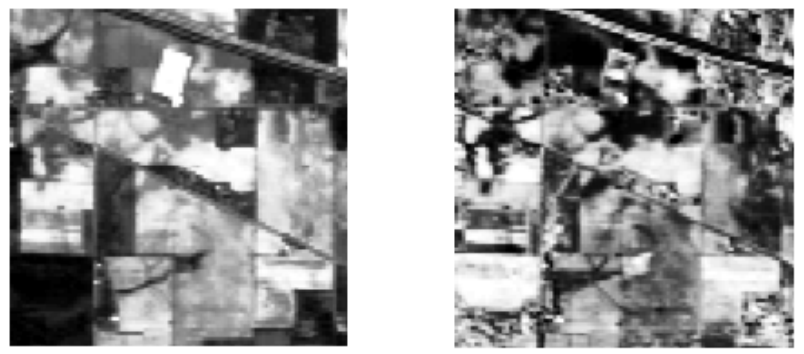

Figure 10. First two PCs of Indian pines hyperspectral image

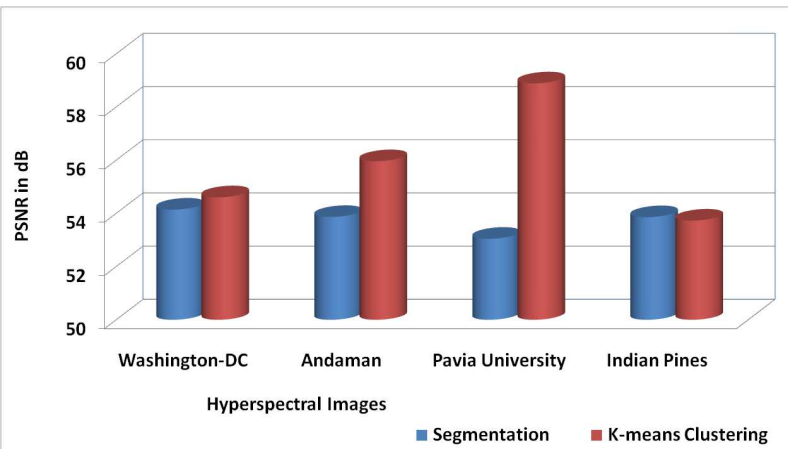

Figure 11. Comparative evaluation of PSNR for four different hyperspectral images using segmentation and k-means clustering.

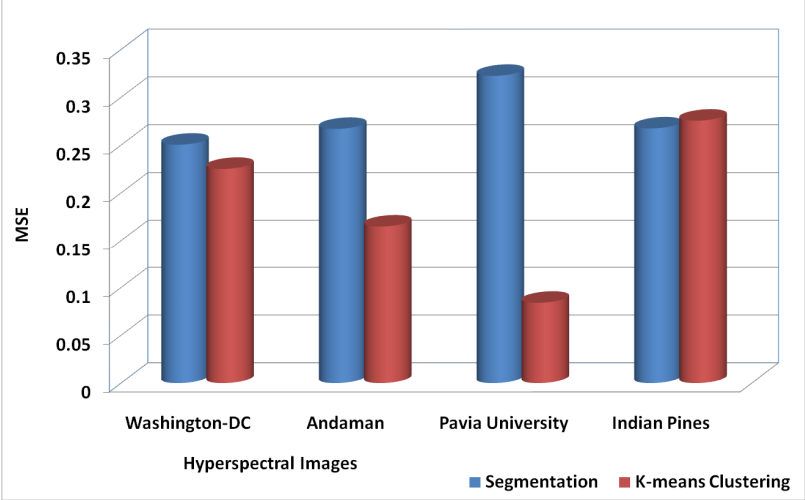

Figure 12. Comparative evaluation of MSE for four different hyperspectral images using segmentation and k-means clustering.

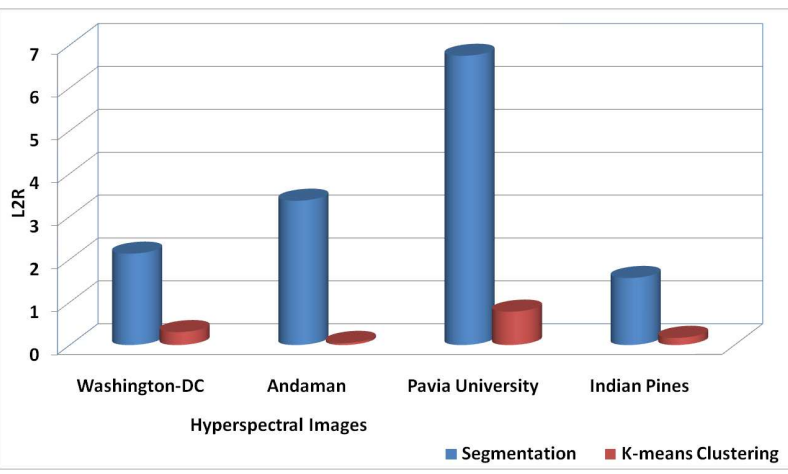

Figure 13. Comparative evaluation of L2RAT for four different hyperspectral images using k-means clustering and segmentation.

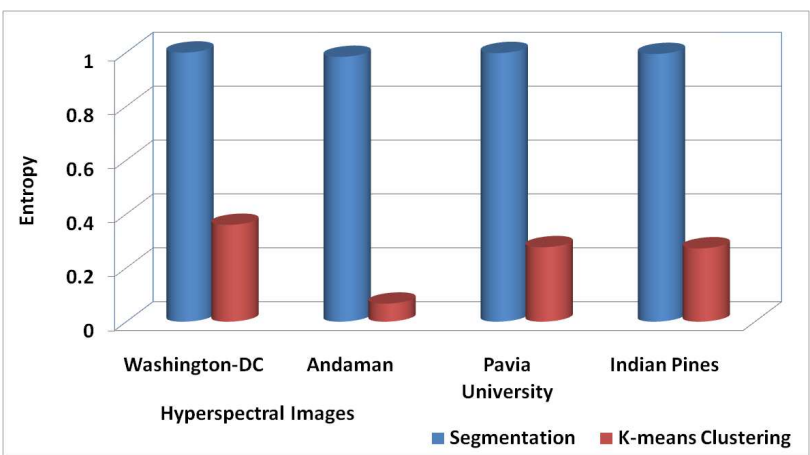

Figure 14. Comparative evaluation of Entropy for four different hyperspectral images using k-means clustering and segmentation.

A portion of Hydice Washington-DC hyperspectral image is show in Figure 3. Figure 4 shows first two Principal Components (PCs) of a portion of Hydice Washington-DC hyperspectral image. Similarly Figure 5, Figure 7 and Figure 9 shows hyperspectral images of other three data sets which were considered for processing. Figure 6, Figure 8 and Figure 10 shows first two PCs of other three data sets which were considered for processing in our experiments. 
Figure 11 shows that PSNR for k-means clustering gives better result in comparison with segmentation for the images of Hydice, IMS1 and Pavia University but gives slightly lower value for Indian pines. Pavia University is having highest geometric resolution of 1.3 meters and it is giving highest PSNR in $\mathrm{dB}$ for $\mathrm{k}-$ means clustering. That means this image is classified more accurately using k-means clustering than segmentation.

Figure 12 show that MSE for K-means clustering gives lesser values compared with segmentation for the images of Hydice, IMS1 and Pavia University but gives slightly higher value for Indian pines. Pavia University is giving lowest MSE for kmeans clustering. That again proves that this image is classified more accurately using k-means clustering than segmentation.

Results from Figure 13 clearly show that energy difference for segmentation is very high for all the images between the original image and segmented image. That means classified image using segmentation is less accurate compared to the $\mathrm{K}$ means clustering for all the images.

If we see results from Figure 14, it is clear that entropy for all the images are higher for segmentation due the simple reason that more randomness is present in the segmented image. In other case, for K-means clustering the randomness is lesser compared with segmented image. Computation of Maximum Squared Error does not show any large variation for all the images while processed with both the techniques hence Maximum Squared Error has been discarded in our metrics evaluation. With these results we can clearly say that quality of classified images can be evaluated using metrics such as PSNR, MSE, L2RAT and Entropy where visual evaluation is not feasible.

\section{CONCLUSION}

We have studied k-means clustering and segmentation for four hyperspectral images to generate metric evaluations. Selected hyperspectral images were of different spectral and geometric resolutions and also they were different in the terms of sensors working platform like Aerial and spaceborn. Dimensionality of hyperspectral data has been reduced using Principle Component Analysis which resulted in reducing the complexity of processing. Metric results from elaborate comparison were generated between k-means clustering and segmentation for all the images by taking PSNR, MSE, L2RAT and Entropy. These parameters clearly indicate that higher geometric resolution image will give highest classification accuracy.

\section{ACKNOWLEDGEMENTS}

The authors are thankful to Director, ISRO Satellite centre, CGM facilities and DGM, ETF-1 for encouraging research activity in centre. The authors wish to thank the following individuals for their technical support, Mr. Uday Raj General manager RRSC south, Mr. Hebbar and Mr. C. J. Jagadesha from RRSC South.

\section{REFERENCES}

Acharjya, P.P., et al., 2012. An Overview on Watershed Transform and Its Consequences, International Journal of Engineering and Innovative Technology (IJEIT), Volume 1, Issue-5.

Bhuvan, NRSC, ISRO, Hyderabad, INDIA, http://bhuvan.nrsc.gov.in/bhuvan_links.php

Chandhok, C., et al., 2011. An Approach to Image Segmentation using K-means Clustering Algorithm, International Journal of Information Technology (IJIT), Volume - 1, Issue-1.

Christophe, E., et al., 2005. Comparison and evaluation of quality criteria for hyperspectral imagery, IS\&T/SPIE 17th Annual Symposium.

Dhanalakshmi, P., et al., 2013. Automatic Segmentation of Brain Tumor using K-Means Clustering and its Area Calculation, International Journal of Advanced Electrical and Electronics Engineering (IJAEEE), 2278-8948, Volume-2, Issue-2.

Gonzalez, R. C., et al., 2009. Digital Image Processing Using $M A T L A B$, Second Edition, Gates mark Publishing.

Green, A.A., et al., 1988. A transformation for ordering multispectral data in terms of image quality with implications for noise removal, IEEE Trans. Geosci. Remote Sens., vol. 26, no. 1 ,pp. 65-74.

https://engineering.purdue.edu/ biehl/MultiSpec/

Kaur, M., et al., 2011. Medical Image Segmentation using Marker Controlled Watershed Transformation, IJCST, Vol. 2, Issue-4.

Phillips, R.D., et al., 2009. An Adaptive Noise-Filtering Algorithm for AVIRIS Data With Implications for Classification Accuracy, IEEE Trans. Geosci. Remote Sens., vol. 47 , no. 9.

Tarabalka Y., et al., 2010. SVM- and MRF-based method for accurate classification of hyperspectral images, IEEE Trans. on Geosci. and Remote Sensing Letters, DOI 10.1109/LGRS.2010.2047711.

Zhang, J., et al., 2001. Classification of hyperspectral data using support vector machine, IEEE, 0-7 803-6725-1. 